\title{
Addressing the Difficulties of Differentiating Members of the Feldspar Solid Solution Series via Electron Backscatter Diffraction
}

\author{
Shawn W. Wallace ${ }^{1}$ and Stuart Wright ${ }^{2}$ \\ 1. EDAX, A Division of Ametek, Mahwah NJ, USA. \\ 2. EDAX, A Division of Ametek, Draper UT, USA. \\ * Corresponding author: Shawn.Wallace@ametek.com
}

Many minerals in the geological realm behave as solid solutions with one of the most common of these being the Feldspar series. Combine this with lower symmetries and it can be difficult to both index and differentiate these minerals. One final complication is that the phase files available for these phases may be for chemical compositions that are much different from the actual sample. This presents many difficulties in indexing the EBSD patterns. Understanding the differences in the patterns with small changes in chemistry and atomic positions can help the analyst get more correct orientations and less noise in geological EBSD data. Using dynamically simulated EBSD patterns, it is possible to model the differences between the members of the solid solutions to come up with strategies to increase the precision and accuracy of indexing routines.

To simulate EBSD patterns for each phase, a base phase file was needed for lattice parameters, space group and atomic positions. A search was performed on the AMCS database [1] for the base phase name. The data was then compiled in a spreadsheet to look at both consistency of lattice parameters with other entries, geologically relevant experimental conditions (pressure/temperature) or natural specimens. The selected phase data were used to create a master pattern file used to simulate individual EBSD patterns [2]. A pattern was then simulated for each phase. On visual inspection, the majority of these simulated patterns appear nearly identical (see Figure 1).

A normalized dot product between these simulated patterns [3] was calculated to quantify how different the patterns are from each other (see Figure 2(a)) in order to better understand indexability and phase differentiation. As a baseline, albite patterns were simulated for orientations between an orientation of $\varphi_{1}=30^{\circ}, \Phi=30^{\circ}, \varphi_{2}=30^{\circ}$ to a second orientation rotated $10^{\circ}$ in $0.1^{\circ}$ steps from the first. This provides a baseline to correlate the normalized dot product with misorientation (see Figure 2(b)). As noted in [3], the normalized dot product correlates well for small misorientations $\left(<1.5^{\circ}\right)$ but not for large. Note that for a conservative estimate of $0.5^{\circ}$ for Hough based indexing angular precision, the corresponding pattern difference metric is 0.95 . This would suggest that when the normalized dot product is less than 0.95 the two phases should be differentiable. Comparing this tolerance value to the table in Figure 2(b) suggests that the phases between the Albite and Sanidine end members should be relatively easily differentiated whereas the phases between Albite and Anorthite would be more difficult but still possible. That said, current methods using the Hough and reflector lists may need careful optimization focused around differences between the phases to consistently achieve reliable results. Another option would be to use chemistry assisted indexing [4], but long collection times may be required to get quantifiable differences in chemistry between some members. Another approach is to use dictionary indexing for characterizing these minerals [5]. While the results shown suggest this would be a good approach, for these low symmetry materials such an approach is computationally intensive especially if a small average misorientation (e.g. $0.5^{\circ}$ ) in the sampling of orientation space is needed for differentiation between phases. 
[1] RT Downs. M Hall-Wallace, American Mineralogist 88 (2003), p. 247.

[2] PG Callahan, M De Graef, Microscopy and Microanalysis, 19 (2013), p. 1255

[3] S Singh, M De Graef, Model. Simul. Mater. Sc. 24 (2016), p. 085013

[4] MM Nowell, SI Wright, J. Microscopy 213 (2004), p. 296.

[5] K Marquardt, et al., American Mineralogist 102 (2017), p. 1843-1855.

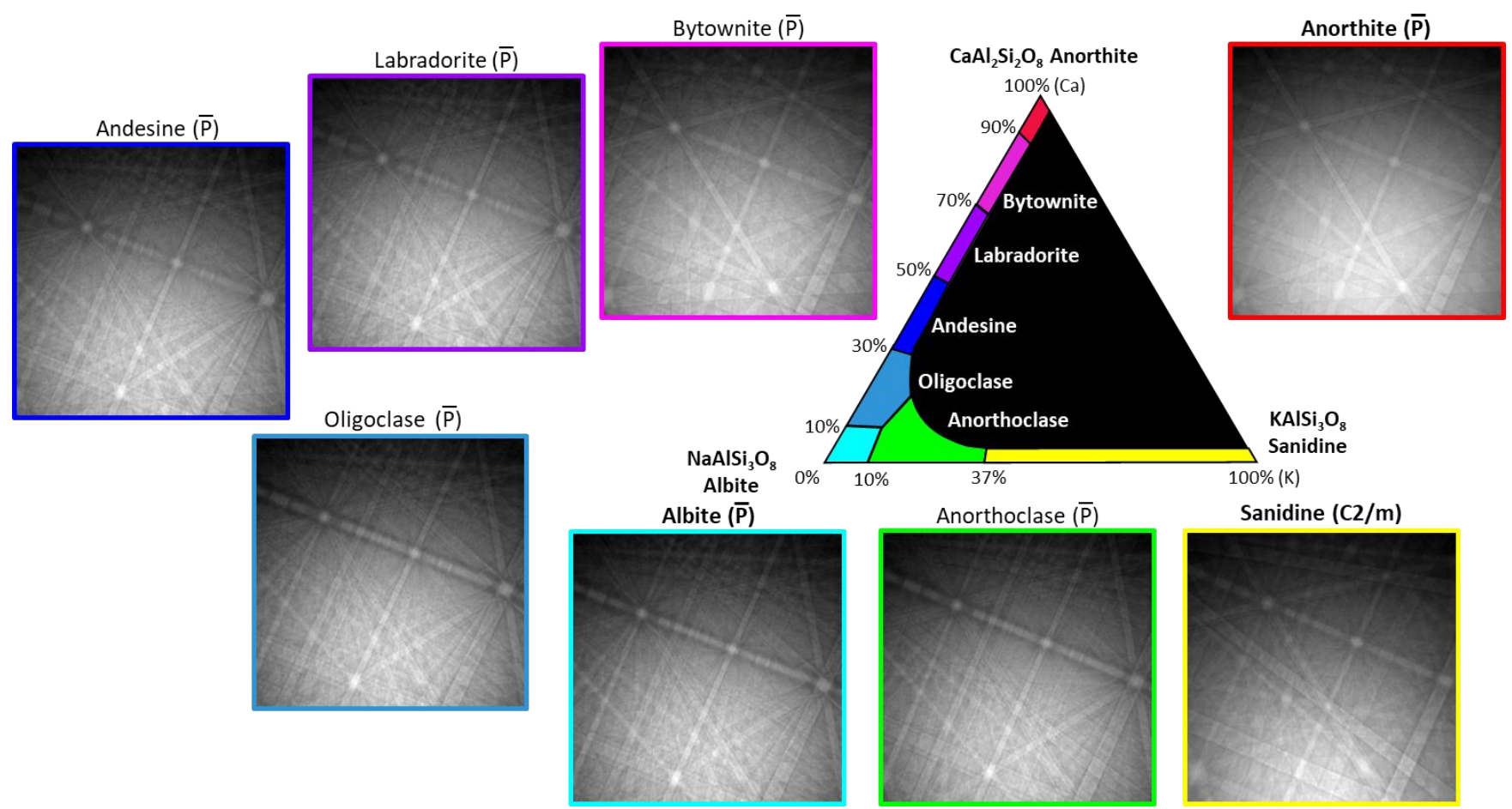

Figure 1. Simulated patterns for members of the Feldspar solid solution series. Ternary diagram adapted from https://www.cefns.nau.edu/geology/naml/Meteorite/Book-GlossaryF.html

\begin{tabular}{|c|c|c|c|c|c|c|c|c|}
\hline $\begin{array}{l}\text { Normalized } \\
\text { Dot Product }\end{array}$ & 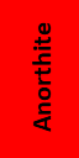 & 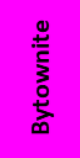 & 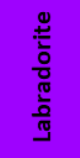 & & 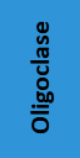 & 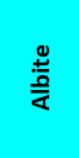 & 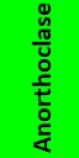 & 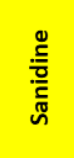 \\
\hline Anorthite & 1 & 0.946 & 0.905 & 0.903 & 0.877 & 0.886 & 0.859 & 0.838 \\
\hline Bytownite & 0.946 & 1 & 0.929 & 0.920 & 0.884 & 0.904 & 0.843 & 0.826 \\
\hline Labradorite & 0.905 & 0.929 & 1 & 0.988 & 0.915 & 0.957 & 0.853 & 0.824 \\
\hline Andesine & 0.903 & 0.920 & 0.988 & 1 & 0.929 & 0.975 & 0.853 & 0.820 \\
\hline Oligoclase & 0.877 & 0.884 & 0.915 & 0.929 & 1 & 0.944 & 0.821 & 0.819 \\
\hline Albite & 0.886 & 0.904 & 0.957 & 0.975 & 0.944 & 1 & 0.838 & 0.820 \\
\hline Anorthoclase & 0.859 & 0.843 & 0.853 & 0.853 & 0.821 & 0.838 & 1 & 0.839 \\
\hline Sanidine & 0.838 & 0.826 & 0.824 & 0.820 & 0.819 & 0.819 & 0.839 & 1 \\
\hline
\end{tabular}

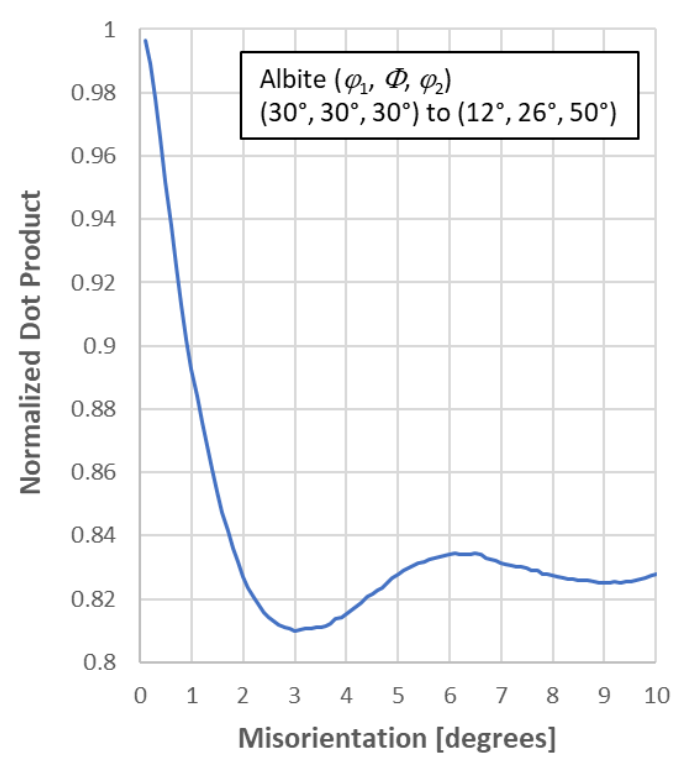

Figure 2. (a) Normalized dot products between pairs of simulated patterns for selected phases in the Feldspar series. (b) Dot product plotted against misorientation for simulated patterns of Albite. 